Article

\title{
Properties of Conductive Polyacrylonitrile Fibers Prepared by Using Benzoxazine Modified Carbon Black
}

\author{
Damiro Ahn ${ }^{1,2}$, Hyun-Jung Choi ${ }^{1}$, Ho-dong Kim ${ }^{2}$ and Sang Young Yeo ${ }^{1, *}$ \\ 1 Technical Textile \& Materials R\&D Group, Korea Institute of Industrial Technology, 143 Hanggaulro, \\ Sangnok-gu, Ansan-si 15588, Korea; ahndamiro94@kitech.re.kr (D.A.); hjchoi@kitech.re.kr (H.-J.C.) \\ 2 Department of Fiber System Engineering, Dankook University, 152 Jukjeon-ro, Suji-gu, Yongin-si 16890, \\ Korea; hodong@dankook.ac.kr \\ * Correspondence: miracle@kitech.re.kr
}

Received: 12 December 2019; Accepted: 1 January 2020; Published: 9 January 2020

\begin{abstract}
Composites of carbon black (CB) and polymers are attractive for producing conductive fibers. Herein, to achieve improved interactions with polymers, the surface of $C B$ was modified to form 4-aminobenzoyl-functionalized carbon black (ABCB), benzoxazine-functionalized carbon black (BZCB), and Ag-anchored carbon black (Ag-ABCB). The surface-modified CBs were characterized by Fourier transform infrared spectroscopy and thermogravimetric analysis, and X-ray photoelectron spectroscopy was utilized to confirm the presence of $\mathrm{Ag}$ in Ag-ABCB. Conductive polyacrylonitrile (PAN) fibers were wet-spun with conductive fillers $(\mathrm{CB}, \mathrm{ABCB}, \mathrm{Ag}-\mathrm{ABCB}$, and $\mathrm{BZCB})$ to investigate the effects of various functional groups on the electrical and mechanical properties. After annealing the conductive PAN fibers, the conductivity and tensile strength greatly increased, whereas the diameter decreased. Notably, the fiber with a BZCB/PAN weight ratio of $12 / 88$ possessed a conductivity of $8.9 \times 10^{-4} \mathrm{~S} / \mathrm{cm}$, and strength of $110.4 \mathrm{MPa}$, and thus the highest conductivity and best mechanical properties in the conductive PAN fiber. These results indicate that the annealed BZCB/PAN fibers have potential applications in the manufacturing of antistatic fabrics.
\end{abstract}

Keywords: polyacrylonitrile; carbon black; wet spinning; conductive fiber; tensile strength

\section{Introduction}

Recently, the convergence of electronic devices and IT technology has advanced the global development of smart textiles with essential core materials made up of conductive fibers. Electrically conductive fibers have various applications depending on the electrical conductivity range; for example, antistatic applications $\left(10^{-5}\right.$ to $\left.10^{0} \mathrm{~S} / \mathrm{cm}\right)$, electric heating $\left(10^{-2}\right.$ to $\left.10^{2} \mathrm{~S} / \mathrm{cm}\right)$, electric wires $\left(>10^{3} \mathrm{~S} / \mathrm{cm}\right)$, wearable sensors $\left(10^{0}\right.$ to $\left.10^{1} \mathrm{~S} / \mathrm{cm}\right)$, and stealth applications $\left(10^{0}\right.$ to $\left.10^{2} \mathrm{~S} / \mathrm{cm}\right)$. It is expected that the demand for electrically conductive fibers will continue to increase in the future [1-5].

There are several methods to fabricate conducting fibers. Some of the established methodologies are the formation of a metal layer on the surface of a non-conducting fiber; adding conducting agents such as carbon black (CB), carbon nanotubes, graphene, $\mathrm{Cu}$, or Ag to polymer chains; and spinning metal fibers. Among these methods, the deposition or plating of conductive material on the surface of a nonconductive fiber is advantageous because the fibers produced tend to exhibit high conductivity and good processability. However, there are also a few disadvantages associated with this, such as weak stabilities toward metal oxidation, susceptibility to friction and wear, and the use of large amounts of non-recyclable chemicals during the manufacturing process. On the other hand, the spinning of conductive materials is not competitive in cost and it remains difficult to industrialize owing to problems with corrosion resistance and processability. However, the method of adding conducting 
agents to polymer chains provides excellent stability and processability, and it has been studied for a long time [6-11].

$\mathrm{CB}$ is a polycrystalline material in which spherical particles are obtained by the incomplete combustion or pyrolysis of hydrocarbons entangle in grape-like clusters. CB is inexpensive and has high dispersibility and processability. It is commercially available as a rubber reinforcing additive, pigment, conductive additive, etc. [12-14]. There are several manufacturing methods for CB/polymer composite fibers, such as solution spinning, melt spinning, electrospinning, and bubbfil spinning. In an earlier study, we reported the fabrication and electrical properties of fibers with functionalized CB added to the PET matrix using melt spinning [15]. However, the melt spinning method has a disadvantage in that the content of the filler in the polymer matrix is limited. Therefore, wet spinning has been commonly used in conductive fiber research because greater amounts of filler can be injected than melt spinning, and dispersion can be achieved in a variety of ways. Besides, it is possible to spin the polymers, which cannot melt, and various studies have been undertaken [16-18].

To spin conductive $\mathrm{CB} /$ polymer composite fibers, the $\mathrm{CB}$ material must reach a percolation threshold while maintaining sufficient dispersibility to pass through the spinning nozzle. Many studies have focused on spinning methods using different types of polymer matrices with CB. However, even when the fibers produced exhibit electrical conductivity, they tend to have poor physical properties because of $\mathrm{CB}$ in the fiber agglomerates. Therefore, it is necessary to develop CB materials with high tensile strength and high electrical conductivity by tailoring its interactions with the polymer to produce versatile fibers [19-23].

In this study, we investigated the electrical and mechanical properties of polyacrylonitrile (PAN) fibers prepared using modified CB materials as conductive fillers. The effects of various surface functional groups on $\mathrm{CB}$, including 4-aminobenzoyl groups, benzoxazine groups, and Ag metal, were compared.

\section{Materials and Methods}

For our studies, we used polyacrylonitrile (PAN, P-30T, Shaoxing Gimel Advanced Materials Technology Co., Ltd., Shaoxing, China) as the polymer matrix and $\mathrm{N}, \mathrm{N}$-dimethylformamide (DMF, 99\%, Daejung Chemical Co., Ltd., Shiheung, Korea) as the solvent. CB (Ketjenblack EC 600JD, AkzoNobel, Amsterdam, Netherlands) was modified using the methods outlined in Figure 1. The 4-aminobenzoic acid ( $\geq 99 \%$, 4-ABAc), sodium borohydride $\left(>98 \%, \mathrm{NaBH}_{4}\right)$, phenol $(99.0-100.5 \%)$, para-formaldehyde (95\%, PFA), and ethanol (EtOH) were obtained from Sigma Aldrich Chemical Inc. (St. Luis, MO, USA), and used without any further treatment. The polyphosphoric acid $\left(83 \% \mathrm{P}_{2} \mathrm{O}_{5}\right.$ assay, $\left.\mathrm{PPA}\right)$, phosphorus pentoxide $\left(98 \%, \mathrm{P}_{2} \mathrm{O}_{5}\right), 1$, 4 -dioxane $(99 \%)$, and silver nitrate $\left(\mathrm{AgNO}_{3}\right)$ were purchased from Daejung Chemical Co., Ltd.

\subsection{CB Modification}

\subsubsection{4-Aminobenzoyl-Functionalized Carbon Black (ABCB)}

We adopted the synthesis method reported by Lee et al. [24] and Choi et al. [15]. In brief, a mixture of 4-ABAc (3.65 mmol), CB (20 g), PPA (1000.0 g), and $\mathrm{P}_{2} \mathrm{O}_{5}(250.0 \mathrm{~g})$ was placed in a $2 \mathrm{~L}$ resin flask equipped with a high-torque mechanical stirrer and a nitrogen inlet and outlet. The reaction was carried out stepwise first at $80^{\circ} \mathrm{C}$ for $6 \mathrm{~h}$; then, at $100^{\circ} \mathrm{C}$ for $10 \mathrm{~h}$; and finally, at $130^{\circ} \mathrm{C}$ for $72 \mathrm{~h}$ under nitrogen atmosphere. The mixture was poured into distilled water, and the resulting powdery product was collected by suction filtration and Soxhlet-extracted with water for 3 days to completely remove the residual reaction medium; and once again, Soxhlet-extracted with ethanol for 1 day to remove the unreacted 4-ABAc. Finally, the obtained mass was freeze-dried under reduced pressure $(0.05 \mathrm{mmHg})$ for 3 days. 


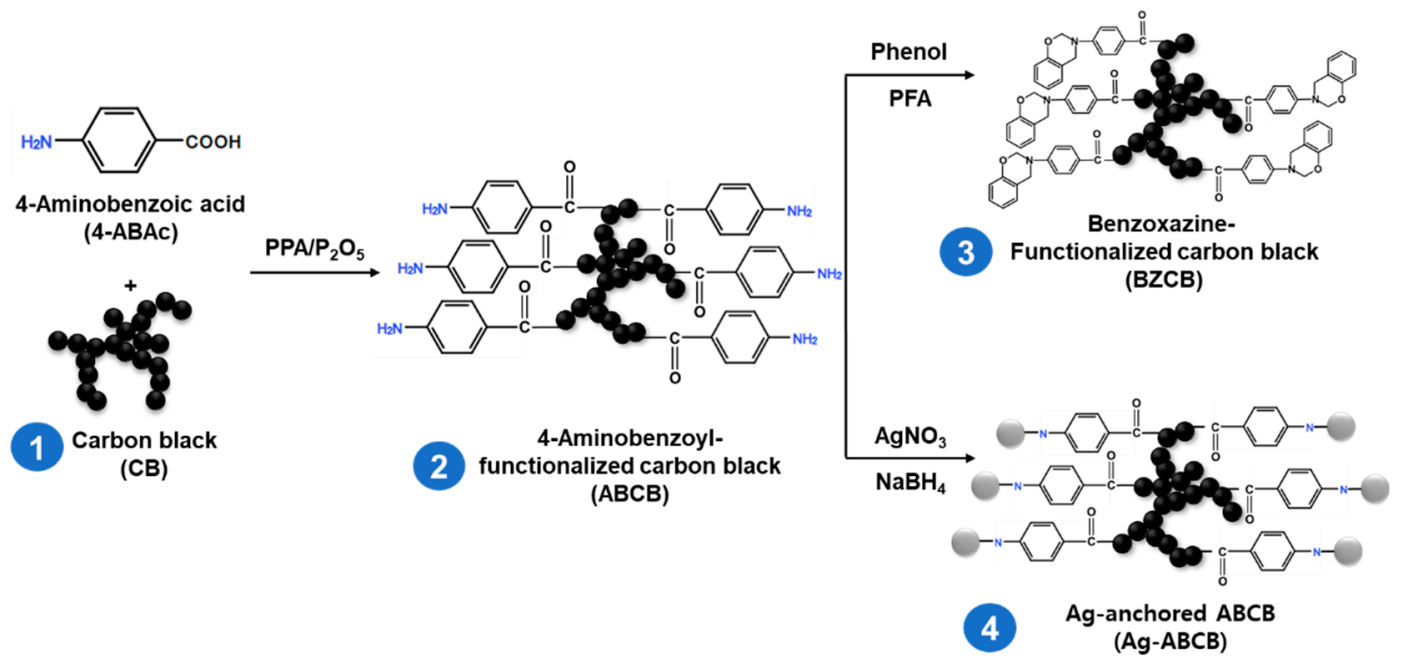

Figure 1. Schematic representation of the carbon black $(\mathrm{CB})$ modification processes.

\subsubsection{Benzoxazine-Functionalized Carbon Black (BZCB)}

BZCB was synthesized following the method of Andreu et al. [25]. ABCB (5 g), phenol (5 g), and PFA $(3.5 \mathrm{~g})$ in a molar ratio of 1:2:4 were refluxed in 1,4-dioxane $(650 \mathrm{~mL})$ under magnetic stirring for 5 days. The resultant powdery product was collected by suction filtration and then Soxhlet-extracted with water for 3 days to completely remove residual reaction medium and unreacted monomers. Finally, the sample was freeze-dried under reduced pressure $(0.05 \mathrm{mmHg})$ for 3 days.

\subsubsection{Ag-Anchored ABCB (Ag-ABCB)}

To prepare $\mathrm{Ag}-\mathrm{ABCB}, \mathrm{ABCB}(10 \mathrm{~g})$ was rapidly dispersed in $\mathrm{AgNO}_{3}$ solution $(0.5 \mathrm{mmol}$ in $\mathrm{EtOH})$. Then, $\mathrm{NaBH}_{4}(0.1 \mathrm{mmol}$ in EtOH) was slowly added drop wise, resulting in the attachment of $\mathrm{Ag}$ to the $\mathrm{ABCB}$ surface $(\mathrm{Ag}-\mathrm{ABCB})$. Finally, the resulting product was washed several times with distilled water and the sample was freeze-dried under reduced pressure $(0.05 \mathrm{mmHg})$ for 3 days.

\subsection{Preparation of Fibers}

The solutions for spinning PAN were prepared by dissolving the $20 \mathrm{wt} \%$ of the polymer in DMF. Then, CB (10 wt \%), ABCB (12 wt \%), BZCB (12 wt \%), or Ag-ABCB (20 wt \%) was dispersed in the polymer solution using a homogenizer (HG-15D, Daihan Scientific Co., Ltd., Wonju, Korea) at $7500 \mathrm{rpm}$ for $30 \mathrm{~min}$ at room temperature. The dispersed solution was allowed to stand for 1 day at room temperature for degassing and then directly used for wet-spinning. The spun fibers were heat-treated in a vacuum oven at $180^{\circ} \mathrm{C}$ for $24 \mathrm{~h}$ under constant tension. Figure 2 shows the schematic of the fiber manufacture processes and the conditions applied for each process.

\subsection{Characterization}

Various spectroscopic techniques were employed to analyze the surface chemical compositions of the modified CBs, which includes Fourier transform infrared (FT-IR) spectroscopy (Spectrum Two, Perkin-Elmer, Waltham, MA, USA) and X-ray photoelectron spectroscopy (XPS, K-alpha, ThermoFisher, Waltham, MA, USA). For the FT-IR measurements, the CBs were pelletized with potassium bromide $(\mathrm{KBr})$ as the diluent and the spectra were collected in the scan range of $500-4000 \mathrm{~cm}^{-1}$. The XPS analysis was conducted in the range of $0-3000 \mathrm{eV}$. Thermogravimetric analysis (TGA) was performed using a thermogravimetric analyzer (Q500, TA Instruments, New Castle, DE, USA) from 25 to $800^{\circ} \mathrm{C}$ at a heating rate of $20^{\circ} \mathrm{C} / \mathrm{min}$ under airflow and nitrogen atmosphere. Field emission-scanning electron microscopy (FE-SEM; SU8000, Hitachi Ltd., Tokyo, Japan) was used to confirm the morphology of the synthesized conductive filler and degree of dispersion in the PAN matrix. All samples were cut after 
pre-treatment with liquid nitrogen. Energy dispersive X-ray spectroscopy (EDX) was measured by Hitachi S-4700.

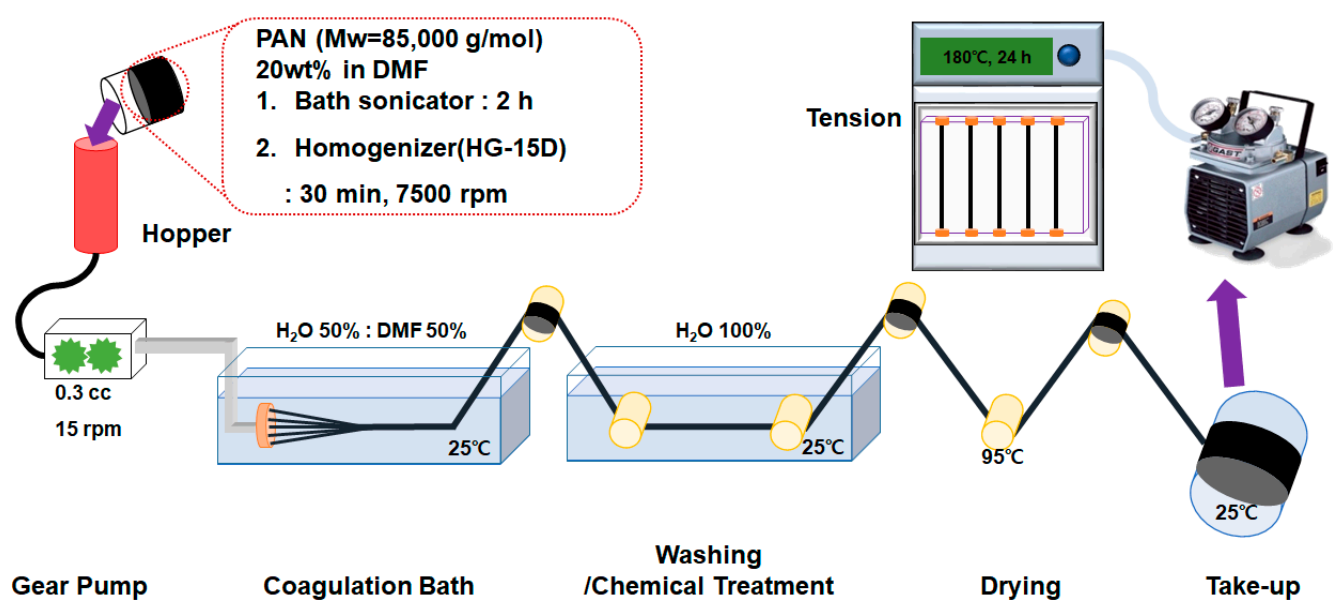

Figure 2. Schematic of the manufacturing processes for conductive fibers.

Electrical conductivity measurements were conducted using various instruments, depending on the magnitude of the conductivity. Fiber-type samples were manufactured by stacking 25 filaments on a glass slide and then covering the filaments with silver paste at intervals of $1 \mathrm{~cm}$. For resistances greater than $10^{-5} \Omega / \mathrm{cm}$, measurements were performed using a Keithley $6517 \mathrm{~B}$ high-resistance meter (Keithley Instruments Inc., Cleveland, OH, USA). For resistances less than $10^{-5} \Omega / \mathrm{cm}$, measurements were performed using a 3280-20F Clamp On HiTester (Hioki, Japan). The measurements were based on the ASTM-D-257 standard test methods, which is a 2-point method. The resistance, $\rho$, was calculated using Equation (1), where $R, A, l$, and $n$ are the measured resistance between the electrodes, the cross-sectional area of the fiber, the distance between the electrodes, and the number of fibers, respectively $[26,27]$. The electrical conductivity of the fiber, $\sigma$, was calculated from the resistance using Equation (2).

$$
\begin{gathered}
\rho(\Omega \cdot \mathrm{cm})=R(\Omega) \times \frac{A\left(\mathrm{~cm}^{2}\right)}{l(\mathrm{~cm})} \times \frac{1}{n} . \\
\sigma(\mathrm{S} / \mathrm{cm})=\frac{1}{\rho(\Omega \cdot \mathrm{cm})} .
\end{gathered}
$$

The Single fiber testing was performed using the Favimat (Textechno, Mönchengladbach, Germany), which measured tensile strength of PAN, CB10/PAN, ABCB12/PAN, BZCB12/PAN, and Ag-ABCB20/PAN fibers. A gauge length of $20 \mathrm{~mm}$ and cross-head speed of $20 \mathrm{~mm} / \mathrm{min}$ were used. The tenacity was the average value of 10 repeated measurements.

\section{Results and Discussion}

\subsection{CB Functionalization}

Figure 1 shows the $\mathrm{CB}$ modification scheme. $\mathrm{ABCB}$ was prepared using $\mathrm{ABAC}$ to functionalize $\mathrm{CB}$ via a Friedel-Crafts acylation reaction. Friedel-Crafts acylation in the PPA $/ \mathrm{P}_{2} \mathrm{O}_{5}$ medium is milder and less corrosive than other functionalization systems. Thus, this reaction does not damage the graphitic framework, allowing the preservation of the intrinsic properties of the graphitic materials [28].

BZCB was synthesized by a Mannich reaction of phenol, para-formaldehyde, and the primary amines of the aminobenzoyl group of $\mathrm{ABCB}$. As BZCB can undergo ring-opening processes in the absence of an initiator or strong and toxic acid catalysts, this material can be easily bonded to PAN via heat treatment $[29,30]$. 
It is known that metal-carbon composites are advantageous for the preparation of high-conductivity composite fibers [15]. Thus, to fabricate a metal-carbon composite (Ag-ABCB), $\mathrm{Ag}$ was attached to the surface of the $\mathrm{ABCB}$ using $\mathrm{AgNO}_{3}$ as a silver precursor and $\mathrm{NaBH}_{4}$ as the reducing agent.

The FT-IR spectra of pristine CB and the modified CBs are presented in Figure 3a. There were no significant functional groups detected on pristine $C B$. However, after modification, the peaks corresponding to various functional groups were observed. For $A B C B$ and $B Z C B$, an apparent keto-carbonyl band was observed near $1651 \mathrm{~cm}^{-1}$, which clearly shows the covalent attachments of the organic moieties to the surface of $\mathrm{CB}$. Other characteristic peaks confirmed the presence of primary amines $\left(\mathrm{N}-\mathrm{H}\right.$ bending vibration at $\left.1603 \mathrm{~cm}^{-1}\right)$ and aromatic amines $(\mathrm{C}-\mathrm{N}$ stretching band at $1311 \mathrm{~cm}^{-1}$ and $\mathrm{N}-\mathrm{H}$ out-of-plane bending vibration at $848 \mathrm{~cm}^{-1}$ ). Additionally, the peaks observed for BZCB at $910-960 \mathrm{~cm}^{-1}$ were not observed for $A B C B$, confirming the presence of a benzoxazine ring in $B Z C B$ [24]. These results indicated that the $C B$ was well modified by the 4-ABAc and benzoxazine group to produce $\mathrm{ABCB}$ and $\mathrm{BZCB}$.
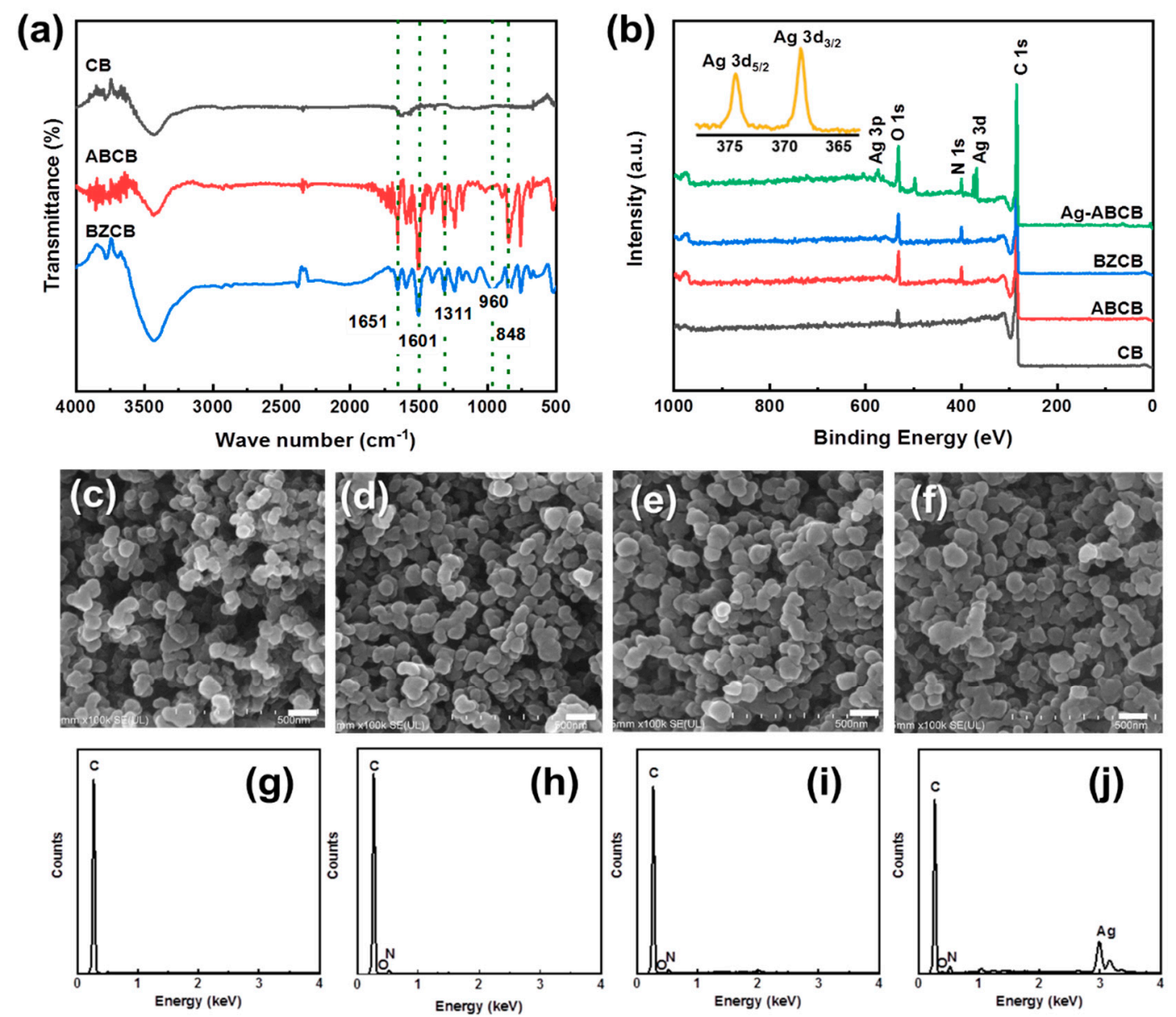

Figure 3. (a) FT-IR and (b) XPS spectra of $C B, A B C B$, and BZCB; SEM images of (c) CB, (d) ABCB, (e) BZCB, and (f) Ag-ABCB. Scale bar is $100 \mathrm{~nm}$. EDX analysis of (g) CB, (h) ABCB, (i) BZCB, and (j) Ag-ABCB.

The XPS spectra of CB before and after modification are shown in Figure $3 \mathrm{~b}$. The CB sample exhibits only two peaks corresponding to $\mathrm{C} 1 \mathrm{~s}$ and $\mathrm{O} 1 \mathrm{~s}$ at binding energies of 297.5 and $545 \mathrm{eV}$, respectively. For $\mathrm{ABCB}$ and $\mathrm{BZCB}$, the $\mathrm{N} 1 \mathrm{~s}$ peak was also apparent (at 403 and $410 \mathrm{eV}$, respectively), and the intensity of the $\mathrm{O} 1$ s peak increased. These results confirm that the $\mathrm{CB}$ surface was successfully modified. Additionally, XPS was used to characterize Ag in Ag-ABCB (Figure 3b). The existence of 
two Ag 3d peaks for Ag-ABCB at binding energies of 368.3 and $374.3 \mathrm{eV}$ with a difference of $6.0 \mathrm{eV}$ confirmed the formation of metallic silver. The standard $\mathrm{Ag} 3 \mathrm{~d}_{5 / 2}$ and $\mathrm{Ag} 3 \mathrm{~d}_{3 / 2}$ binding energies of pure silver are 368.1 and $374.1 \mathrm{eV}$, respectively, and the shift of the $\mathrm{Ag} 3 \mathrm{~d}$ peaks of $\mathrm{Ag}-\mathrm{ABCB}$ to lower binding energies may be due to the electron transfer between the Ag nanoparticles and $C B$ [31].

SEM images show the morphologies of $\mathrm{CB}, \mathrm{ABCB}, \mathrm{BZCB}$, and $\mathrm{Ag}-\mathrm{ABCB}$. Agglomerated particle sizes of approximately $60-70,70-80$, and $70-80 \mathrm{~nm}$ were determined for the $C B, A B C B$, and $B Z C B$ respectively (Figure $3 c-e$ ). The particle size gradually increased in the order $C B<A B C B<B Z C B$ because of 4-ABAc and benzoxazine functionalization. The Figure $3 g-j$ shows the EDX of conductive fillers. The pristine $\mathrm{CB}$ showed a $\mathrm{C}$ peak and after the functionalization 4-ABAc; $\mathrm{O}$ and $\mathrm{N}$ peaks newly emerged. The benzoxazine functionalized showed $\mathrm{C}, \mathrm{O}$, and $\mathrm{N}$ peaks from $\mathrm{CB}$ and the benzoxazine group. In the Ag, anchored $\mathrm{ABCB}$ and $\mathrm{Ag}$ peaks appeared. These results strongly indicate the functionalization of the 4-aminobenzoyl group and benzoxazine group, and attachment of Ag particle on the $\mathrm{ABCB}$. Besides, the bright and white parts indicated by the arrows showed that the partially aggregated silver nanoparticles were well loaded to the $A B C B$ surface (Figure $3 f$ ). The inset in Figure $3 f$ shows Ag peaks from Ag-ABCB nanocomposites, visually showing the effective attachment of the $\mathrm{Ag}$ nanoparticles to the $A B C B$ 's surface.

\subsection{Thermal Properties of Modified CB}

The TGA thermograms of CB and the modified CBs are shown in Figure 4. Thermal decomposition of the materials upon heating was examined under air (Figure 4a) and nitrogen atmospheres (Figure 4b). The thermo-oxidative stabilities of the samples were demonstrated by the thermograms in air, as shown in Figure 4a. Pristine CB showed no weight change until approximately $620^{\circ} \mathrm{C}$, and then the weight decreased rapidly. However, in the cases of $\mathrm{ABCB}$ and $\mathrm{BZCB}$, primary thermal decomposition occurred at 570 and $605{ }^{\circ} \mathrm{C}$ with secondary thermal decomposition occurring at 670 and $698^{\circ} \mathrm{C}$, respectively. The difference between the pyrolysis behavior of pristine $\mathrm{CB}$ and the modified CBs in the air is likely due to the organic moieties covalently attached to the surfaces of the modified CBs, which undergo thermal decomposition at lower temperatures, observed as the primary thermal decomposition process. Analogous to transformations observed during carbonization, during BZCB pyrolysis, the benzoxazine groups form aromatic rings that can react with defects on the $C B$ surface. This process inhibits thermal decomposition during high-temperature charring, and thus improves the thermal stability of BZCB compared with those of the other modified CBs [32]. The char yield of Ag-ABCB at $800{ }^{\circ} \mathrm{C}$ in air was approximately $13 \mathrm{wt} \%$, whereas those of the other samples showed values close to $0 \mathrm{wt} \%$. The residual amount of $\mathrm{Ag}-\mathrm{ABCB}$ at $800{ }^{\circ} \mathrm{C}$ should be related to the $\mathrm{Ag}$ particles, and this amount was $13 \mathrm{wt} \%$. This is a strong indication that the $\mathrm{Ag}$ particles were successfully attached to the surface of the $\mathrm{ABCB}$ and that the residual char yield at $800{ }^{\circ} \mathrm{C}$ was due to the amount of $\mathrm{Ag}$ particle loaded [15].

As shown in the TGA thermograms obtained under a nitrogen atmosphere (Figure $4 \mathrm{~b}$ ), CB displayed linear weight loss behavior, with a char amount of $97.8 \mathrm{wt} \%$ at $800^{\circ} \mathrm{C}$. In contrast, owing to their organic functional groups, $\mathrm{ABCB}$ and $\mathrm{BZCB}$ exhibited weight losses in nitrogen starting at $450-500{ }^{\circ} \mathrm{C}$, with char amounts at $800{ }^{\circ} \mathrm{C}$ of 65.8 and $84.5 \mathrm{wt} \%$, respectively. The difference in the amounts of char for these samples supports the speculation that BZCB may fix the defects on the surface of $\mathrm{CB}$ and prevent $\mathrm{CB}$ thermal decomposition during charring. Additionally, the char amount of $\mathrm{Ag}-\mathrm{ABCB}$ at $800{ }^{\circ} \mathrm{C}(75.8 \mathrm{wt} \%)$ was approximately $10 \mathrm{wt} \%$ higher than that of $\mathrm{ABCB}$. This difference is similar to that observed in the air atmosphere, suggesting that it corresponds to the amount of $\mathrm{Ag}$ particles in Ag-ABCB.

\subsection{Electrical Properties of Modified CB/PAN Fibers}

The electrical conductivities of the modified CB/PAN fibers are shown in Figure 5. The conductivity values were calculated using Equation (2) using the resistances of the fibers measured as-spun (Figure 5a) and annealed fiber (Figure $5 b$ ). The sample names indicate the type and content of CB material in the fiber; for example, the fiber with $10 \mathrm{wt} \%$ pristine $\mathrm{CB}$ as filler is denoted by CB10/PAN. As shown in 
Figure $5 \mathrm{a}$, the $\mathrm{CB} / \mathrm{PAN}$ and $\mathrm{ABCB} / \mathrm{PAN}$ fibers show no change in electrical conductivity, even at the highest filler content. However, BZCB12/PAN and Ag-ABCB20/PAN exhibited significant electrical conductivity values of $1.9 \times 10^{-6}$ and $1.4 \times 10^{-8} \mathrm{~S} / \mathrm{cm}$, respectively. Even at the same filler content, the electrical conductivity of BZCB12/PAN is higher than that of ABCB12/PAN. Various studies have reported the use of benzoxazine resins as a noncovalent dispersant [32]. Thus, modification of the $C B$ surface with benzoxazine groups may weaken the van der Waals forces between $\mathrm{CB}$ particles, which can improve dispersibility and enhance electrical conductivity. Moreover, the Ag-ABCB/PAN fibers exhibit enhanced electrical conductivity because the Ag particles have a higher electrical conductivity than CB [33].

(a)

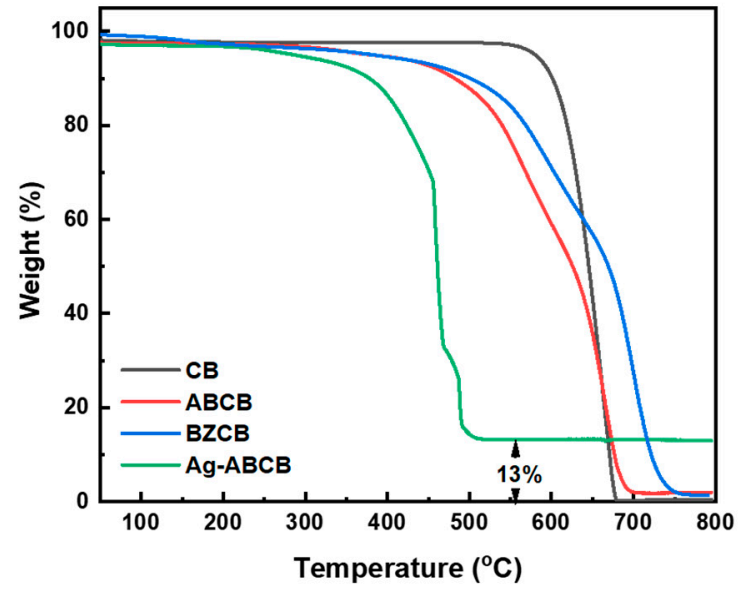

(b)

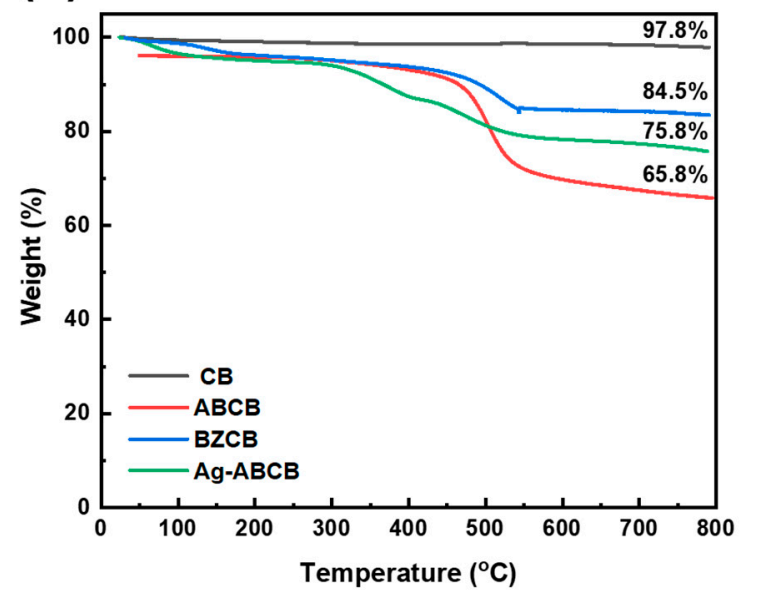

Figure 4. TGA thermograms of modified CBs in (a) air and (b) $\mathrm{N}_{2}$.

(a)

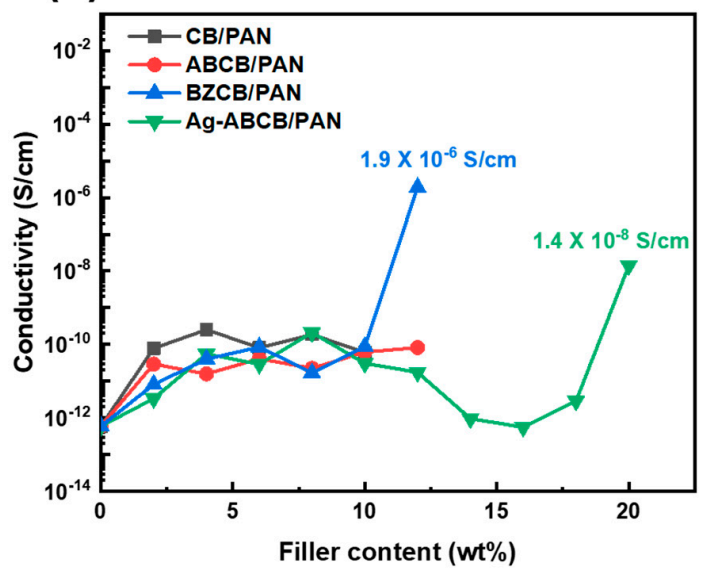

(b)

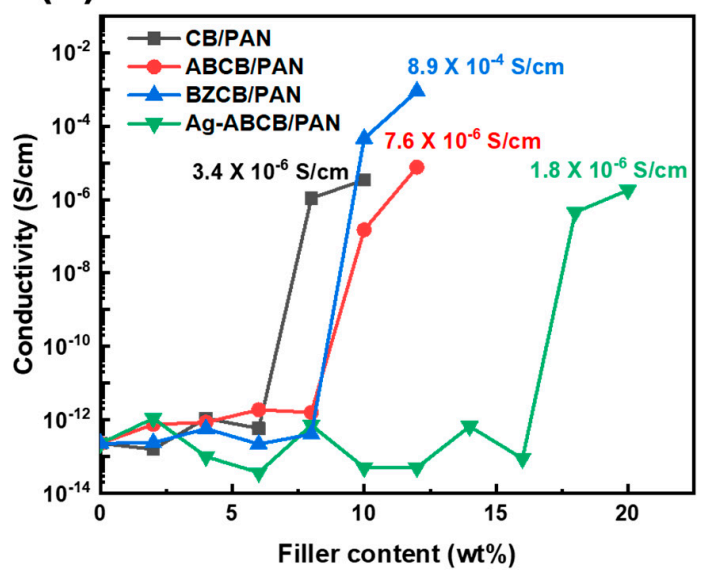

Figure 5. Electrical conductivities of PAN fibers with different contents of CB and modified CBs (a) as-spun and (b) annealed fiber.

As shown in Figure 5b, the annealed CB10/PAN, ABCB12/PAN, BZCB12/PAN, and Ag-ABCB20/PAN fibers exhibit maximum electrical conductivity values of $3.4 \times 10^{-6}, 7.6 \times 10^{-6}$, $8.9 \times 10^{-4}$, and $1.8 \times 10^{-6} \mathrm{~S} / \mathrm{cm}$, respectively. After annealing, all the fibers exhibited a percolation threshold; i.e., the point at which the electrical conductivity sharply increased. It is known that annealing causes phase coarsening to occur, which will drive the $\mathrm{CB}$ nanoparticles to self-assemble into a nanoparticle network, making the $\mathrm{CB} /$ polymer composites conductive. The electrical conductivities of the BZCB/PAN and Ag-ABCB/PAN fibers, which exhibited electrical conductivity as-spun fiber, did not increase significantly. The formation of the $\mathrm{CB}$ conductive network results in strong interactions between the $\mathrm{CB}$ particles, and thus, the morphologies of the $\mathrm{CB} /$ composites are fixed by this network 
structure. However, this effect is less prominent when it occurs at a CB content above the percolation threshold [34,35].

\subsection{Effect of Annealing on Fiber Diameter}

Figure 6 and Table 1 compares the mean diameters of the as-spun and annealed PAN fibers with various CB fillers, as determined from surface SEM images. The chosen content for each filler corresponded to the fibers that exhibited the most pronounced characteristics. The diameters of all the fibers decreased after the annealing process. Especially, the diameters of the as-spun fiber were PAN $53.9 \mu \mathrm{m}$ and Ag-ABCB20/PAN $43.6 \mu \mathrm{m}$, but the diameters of the annealed fibers largely decreased, with values of PAN $41.4 \mu \mathrm{m}$ and Ag-ABCB20/PAN $34.1 \mu \mathrm{m}$. In general, when fibers are annealed, the diameter decreases owing to heat shrinkage [36]. As shown in Figure 6, the diameter reduction ratios of the fibers reinforced with fillers were lower than that of the as-spun PAN fiber. Even though the filler content was the same, the diameter reduction ratio of BZCB12/PAN was lower than that of ABCB12/PAN. This was due to the evolution of annealing by-products of different volatilities and pore generation through the polymeric matrix. As shown in Figure 7, when BZCB is heated, the benzoxazine group can undergo a ring-opening reaction, allowing the formation of covalent bonds or hydrogen bonds with the nitrile groups of PAN. It is reasonable to expect the formation of such bonds to alter the diameter owing to heat shrinkage of the fiber; therefore, the diameter reduction ratio of BZCB12/PAN decreased [30,31].
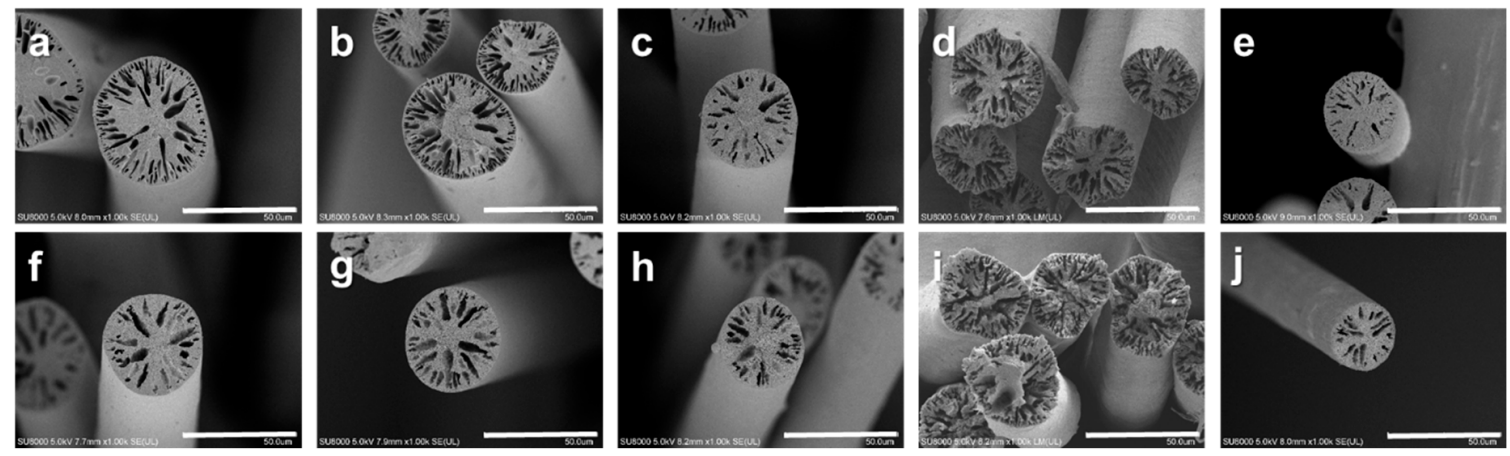

Figure 6. SEM images of as-spun and annealed fibers. As-spun fibers: (a) PANB, (b) CB10/PAN, (c) ABCB12/PAN, (d) BZCB12/PAN, and (e)Ag-ABCB20/PAN. And annealed fiber: (f) PANB, (g) CB10/PAN, (h) ABCB12/PAN, (i) BZCB12/PAN, and (j) Ag-ABCB20/PAN.

Table 1. Diameters of as-spun and annealed fibers.

\begin{tabular}{cccccc}
\hline \multirow{2}{*}{ Sample } & \multicolumn{5}{c}{ Diameter $(\mu \mathrm{m})$} \\
\cline { 2 - 5 } & PAN & CB10/PAN & ABCB12/PAN & BZCB12/PAN & Ag-ABCB20/PAN \\
\hline As-spun Fiber & $53.9 \pm 1.3$ & $48.5 \pm 1.3$ & $43.6 \pm 1.0$ & $39.9 \pm 0.5$ & $43.6 \pm 0.9$ \\
Annealed Fiber & $41.4 \pm 2.3$ & $41.9 \pm 0.4$ & $36.7 \pm 1.5$ & $36.2 \pm 0.6$ & $34.1 \pm 0.5$ \\
\hline
\end{tabular}

\subsection{Mechanical Properties of Modified CB/PAN Fibers}

The uniaxial stress-strain curves of PAN fiber changed with annealing and varying CBs surface are shown in Figure 8. The PAN fiber and the composite fibers containing the carbon black additives increased the tenacity of the annealed fibers compared to the as-spun fibers. In particular, the tenacity (110.4 MPa) of the BZCB12/PAN annealed fiber was increased almost four times higher than that (33.9 $\mathrm{MPa}$ ) of the as-spun fiber. In general, it is known that the tensile strengths of fibers increase after annealing owing to the decrease in the fiber diameter caused by heat shrinkage as well as a decrease in the defects in the fiber introduced during the wet-spinning process. These phenomena explain the increase in the strength from 32.9 MPa to 49.3 MPa of the annealed PAN fiber [37]. 

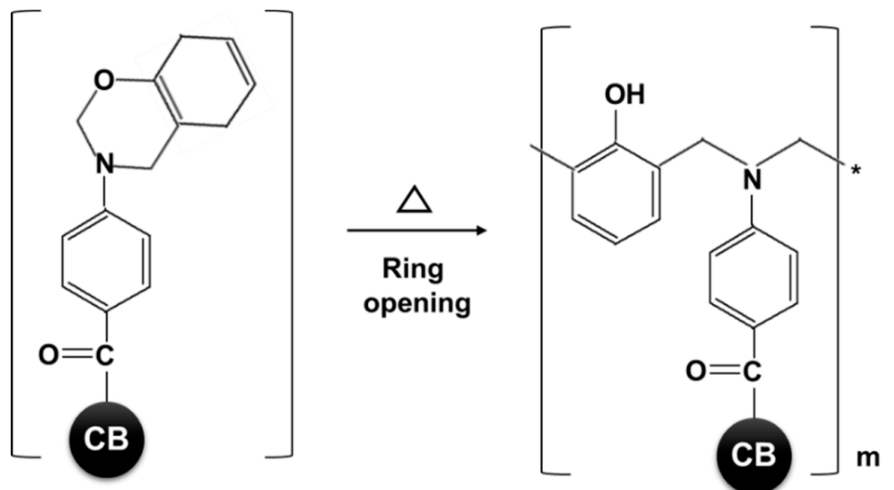

Figure 7. Schematics of ring-opening reaction of the benzoxazine groups in BZCB during annealing.

(a)

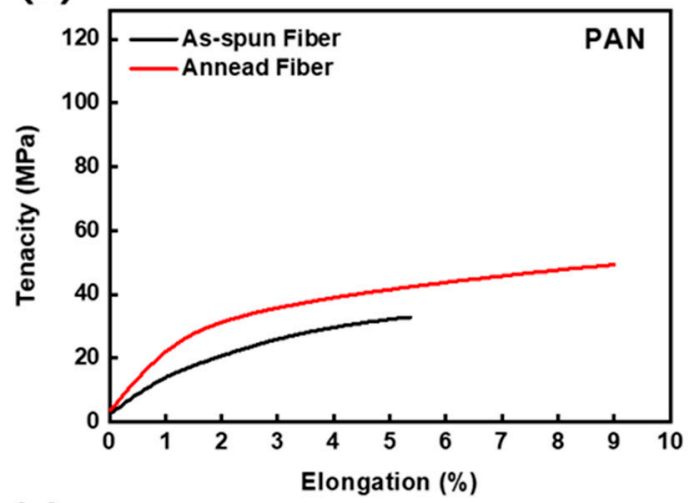

(c)

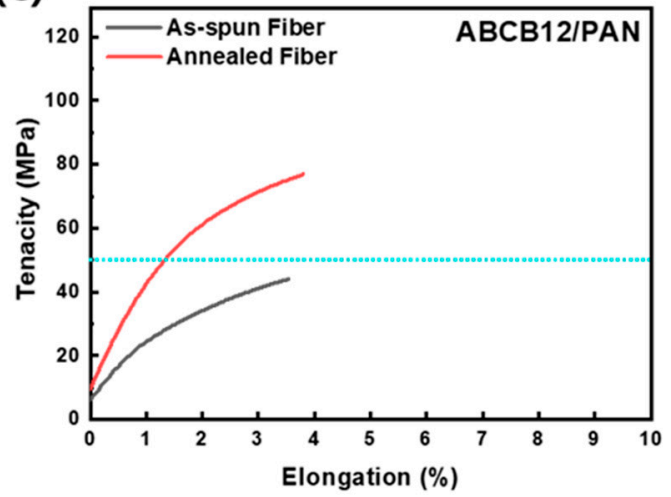

(e)

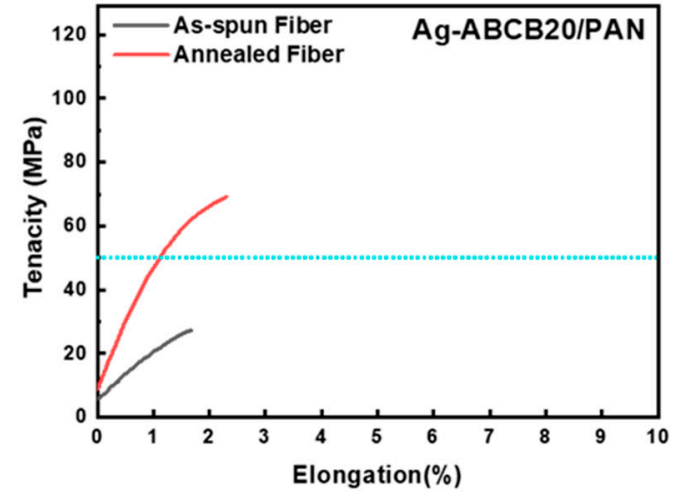

(b)

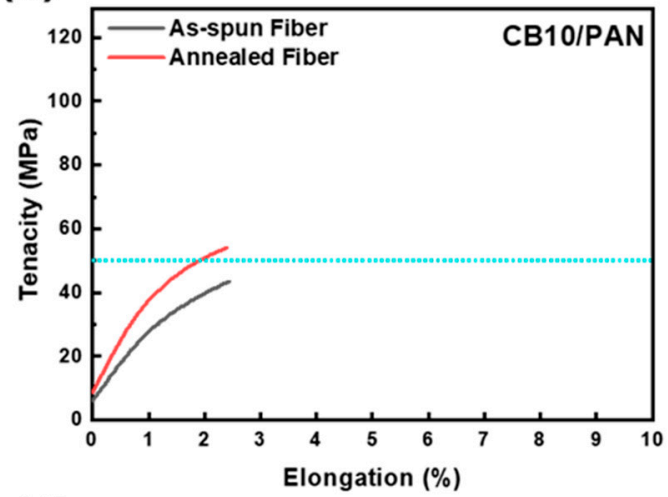

(d)

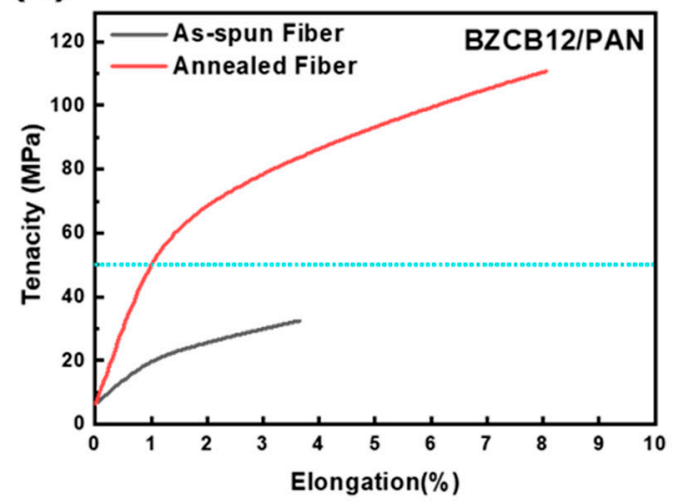

(f)

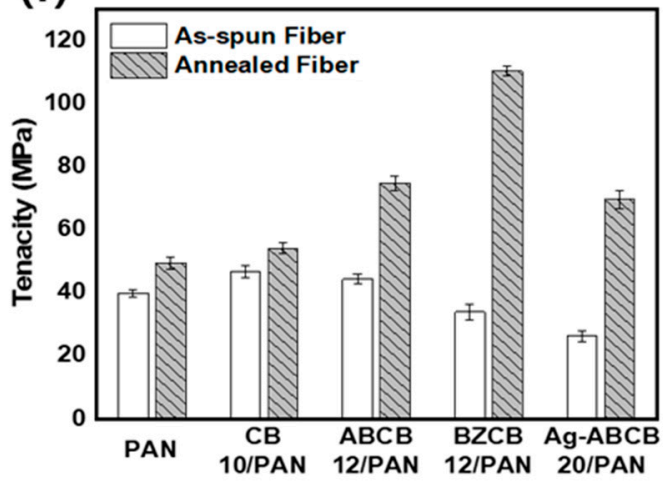

Figure 8. Tensile properties of as-spun PAN fiber and annealed PAN fibers at $180{ }^{\circ} \mathrm{C}$ : (a) PAN, (b) CB10/PAN, (c) ABCB12/PAN, (d) BZCB12/PAN, and (e) Ag-ABCB20/PAN; (f) tenacity of the as-spun and annealed fibers. The sky blue line shows annealed PAN fiber with $49.3 \mathrm{MPa}$ tenacity. 
The mechanical property changes after the annealing process are discussed in the sense of the difference in the chemistry of the CBs surface. The general tendency is that the stress level increases by the addition of $\mathrm{CBs}$, which play the role of reinforcement. The same result was obtained for all the fibers with CBs and was stronger than the PAN fiber. The effects of reinforcement, such as elongation, modulus, and strength of the organic modified CBs, were higher than fiber with inorganic modified CBs. Especially, the reinforcement effect of CBs with organic functional groups was a noticeable elongation of the fiber. It means that the tensile properties of the fiber might have a positive gain from the CBs surface if the interface between the CBs and fiber was secured. In particular, the tensile properties of BZCB12/PAN were the highest after annealing, which, as mentioned above, may be explained by the formation of bonds between the PAN fiber and the filler.

\section{Conclusions}

In the present study, CB was modified with 4-aminobenzoyl groups, benzoxazine groups, or Ag nanoparticles. FT-IR and XPS analyses confirmed the presence of organic functional groups and Ag on the modified CBs. Furthermore, TGA revealed that BZCB showed high thermal stability and indirectly confirmed the presence of Ag particles in Ag-ABCB. Using CB and the modified CBs as fillers, $\mathrm{CB} / \mathrm{PAN}$ and modified $\mathrm{CB} / \mathrm{PAN}$ composite fibers were successfully produced. The electrical and mechanical properties of the composite fibers depend on the surface functional groups on $\mathrm{CB}$. Both the electrical conductivities and tensile strengths of the PAN fibers increased after annealing. After annealing, BZCB12/PAN had the highest electrical conductivity and tensile strength $\left(8.9 \times 10^{-4} \mathrm{~S} / \mathrm{cm}\right.$ and 110.4 MPa, respectively). Furthermore, the fiber diameters decreased upon annealing, and the diameter reduction ratio was lowest for BZCB12/PAN. Owing to the benzoxazine groups in BZCB, the annealing process led to bond formation between PAN and BZCB in the BZCB/PAN composite fiber. As a result, BZCB12/PAN exhibited the best properties among the manufactured conductive fibers.

Author Contributions: D.A., H.-d.K. and S.Y.Y. constructed the original idea according to the concept of analysis; D.A. and H.-J.C. conducted experiments; D.A. carried out the characterizations; D.A. wrote the manuscript; H.-d.K. and S.Y.Y. confirmed the experimental results and discussed with D.A. All authors contributed and commented on this manuscript. All authors have read and agreed to the published version of the manuscript.

Funding: This research was funded by Ministry of Trade, Industry and Energy: 10048884.

Acknowledgments: This research was financially supported by Ministry of Trade, Industry and Energy of Republic of Korea (10048884).

Conflicts of Interest: The authors declare no conflict of interest.

\section{References}

1. Zeng, W.; Shu, L.; Li, Q.; Chen, S.; Wang, F.; Tao, X.M. Fiber-Based Wearable Electronics: A Review of Materials, Fabrication, Devices, and Applications. Adv. Mater. 2014, 26, 5310-5336. [CrossRef] [PubMed]

2. Lancos, L.; Thronickle, W.; Ristol, S. Smart Fabrics White Paper; Atos: Bezons, French, 2017; pp. 1-20.

3. Silva, T.F.; Menezes, F.; Montagna, L.S. Preparation and characterization of antistatic packaging for electronic components based on poly(lactic acid)/carbon black composites. J. Appl. Polym. 2019, 136, 47273-47280. [CrossRef]

4. Abbasi, H.; Antunes, M.; Velasco, J.I. Recent advances in carbon-based polymer nanocomposites for electromagnetic interference shielding. Prog. Mater. Sci. 2019, 103, 319-373. [CrossRef]

5. Lu, H.; Liu, S.; Zhang, Y.; Huang, Y. Nitrogen-Doped Carbon Polyhedra Nanopapers: An Advanced Binder-Free Electrode for High-Performance Supercapacitors. ACS Sustain. Chem. Eng. 2019, 7, 5240-5248. [CrossRef]

6. Dias, T. Electronic Textiles; Woodhead Publishing: Sawston, UK, 2015; pp. 1-158.

7. Kim, Y.; Zhu, J.; Yeom, B.; Prima, M.D.; Su, X.; Kim, J.G. A stretchable nanoparticle conductors with self-organized conductive pathways. Nature 2013, 500, 59-63. [CrossRef]

8. Youssefi, M.; Fanaei, E.; Shanbeh, M. Strain Sensors Based on Electroless Ni-P Plated Polyester Woven Fabrics. Fibers Polym. 2019, 20, 562-568. [CrossRef] 
9. Tang, C.; Long, G.; Hu, X.; Wong, K.; Lau, W.; Fan, M. Conductive polymer nanocomposites with hierarchical multi-scale structures via self-assembly of carbon-nanotubes on graphene on polymer-microspheres. Nanoscale 2014, 6, 7877-7888. [CrossRef]

10. Yadav, M.; Ahmad, S.; Chiu, F.-C. Graphene oxide dispersed polyvinyl chloride/alkyd green nanocomposite film: Processing and physico-mechanical properties. J. Ind. Eng. Chem. 2018, 68, 246-256. [CrossRef]

11. Yadav, M.; Rhee, K.Y.; Park, S.J. Synthesis and characterization of graphene oxide/carboxymethylcellulose/ alginate composite blend films. Carbohydr. Polym. 2014, 110, 18-25. [CrossRef]

12. Donnet, J.B.; Bansal, R.C.; Wang, M.J. Carbon Black: Science and Technology; Marcel Decker: New York, NY, USA, 1993; pp. 1-461.

13. Sanders, I.J.; Peeten, T.L. Carbon Black: Production, Properties, and Uses; Nova Science Publishers: New York, NY, USA, 2011; pp. 1-293.

14. Huang, J.C. Carbon black filled conducting polymers and polymer blends. Adv. Polym. Technol. 2002, 21, 299-313. [CrossRef]

15. Choi, H.-J.; Ahn, D.; Lee, S.; Yeo, S.Y. Electrical properties of silver-attached amine functionalized carbon black/polyethylene terephthalate fibers prepared by melt-spinning. Polymers 2019, 11, 1611. [CrossRef]

16. Hu, C.C.; Chang, S.S.; Lian, N.Y. Preparation and characterization of carbon black/polybutylene terephthalate/polyethylene terephthalate antistatic fiber with sheath-core structure. J. Text. Inst. 2016, 107, 976-984. [CrossRef]

17. Nakamura, S.; Saito, K.; Sawa, G.; Kitagawa, K. Percolation threshold of carbon black-polyethylene composites. Jpn. J. Appl. Phys. 1997, 36, 5163-5168. [CrossRef]

18. Huang, Z.M.; Zhang, Y.Z.; Kotaki, M.; Ramakrishna, S. A review on polymer nanofibers by electrospinning and their applications in nanocomposites. Compos. Sci. Technol. 2006, 63, 2223-2253. [CrossRef]

19. Pokharel, P.; Xiao, D.; Erogbogbo, F.; Keles, O. A hierarchical approach for creating electrically conductive network structure in polyurethane nanocomposites using a hybrid of graphene nanoplatelets, carbon black and multi-walled carbon nanotubes. Compos. Part B Eng. 2019, 161, 169-182. [CrossRef]

20. Leng, J.S.; Huang, W.M.; Lan, X.; Liu, Y.J.; Du, S.Y. Significantly reducing electrical resistivity by forming conductive $\mathrm{Ni}$ chains in a polyurethane shape-memory polymer/carbon-black composite. Appl. Phys. Lett. 2008, 92, 204101-204103. [CrossRef]

21. Chung, K.T.; Sabo, A.; Pica, A.P. Electrical permittivity and conductivity of carbon black-polyvinyl chloride composites. J. Appl. Phys. 1982, 53, 6867-6879. [CrossRef]

22. O'Farrell, C.P.; Gerspacher, M.; Nikiel, L. Carbon black dispersion by electrical measurements. Kautsch. Gummi Kunstst. 2000, 53, 701-710.

23. Choi, H.-J.; Kim, M.S.; Ahn, D.; Yeo, S.Y.; Lee, S. Electrical percolation threshold of carbon black in a polymer matrix and its application to antistatic fiber. Sci. Rep. 2019, 9, 6338-6350. [CrossRef]

24. Lee, H.J.; Han, S.W.; Kwon, Y.D.; Tan, L.S.; Baek, J.B. Functionalization of multi-walled carbon nanotubes with various 4-substituted benzoic acids in mild polyphosphoric acid/phosphorous pentoxide. Carbon 2008, 46, 1850-1859. [CrossRef]

25. Andreu, R.; Espinosa, M.A.; Galia, M.; Diz, V.C.; Ronda, J.C.; Reina, J.A. Synthesis of Novel Benzoxazines Containing Glycidyl Groups: A Study of the Crosslinking Behavior. J. Polym. Sci. Part A 2006, 44, 1529-1540. [CrossRef]

26. Lim, T.H.; Lee, S.H.; Yeo, S.Y. Highly conductive polymer/metal/carbon nanotube composite fibre prepared by the melt-spinning process. Text. Res. J. 2017, 87, 593-606. [CrossRef]

27. Mazinani, S.; Ajji, A.; Dubois, C. Structure and properties of melt-spun PET/MWCNT nanocomposite fibers. Polym. Eng. Sci. 2010, 50, 1956-1968. [CrossRef]

28. Seo, J.M.; Tan, L.S.; Baek, J.B. Defect/Edge-selective functionalization of carbon materials by direct friedel-crafts acylation reaction. Adv. Mater. 2017, 29, 1606317-1606325. [CrossRef] [PubMed]

29. Kao, T.H.; Chen, J.K.; Cheng, C.C.; Su, C.I.; Chang, F.C. Low-surface-free-energy polybenzoxazine/polyacrylonitrile fibers for biononfouling membrane. Polymer 2013, 54, 258-268. [CrossRef]

30. Ishida, H.; Agag, T. Handbook of Benzoxazine Resins; Elsevier: Amsterdam, The Netherlands, 2011; pp. 1-712.

31. Chook, S.W.; Chia, C.H.; Zakaria, S.; Ayob, M.K.; Chee, K.L.; Huang, N.M.; Neoh, H.M.; Lim, H.N.; Jamal, R.; Rahman, R.M.F.R.A. Antibacterial performance of Ag nanoparticles and AgGO nanocomposites prepared via rapid microwave-assisted synthesis method. Nanoscale Res. Lett. 2012, 7, 541-548. [CrossRef] 
32. Mahadik-Khanolkar, S.; Donthula, S.; Sotiriou-Leventis, C.; Leventis, N. Polybenzoxazine aerogels. 1.high-yield room-temperature acid catalyzed synthesis of robust monoliths, oxidative aromatization, and conversion to microporous carbons. Chem. Mater. 2014, 26, 1303-1317. [CrossRef]

33. Wang, C.F.; Kuo, S.W.; Lin, C.H.; Chen, H.G.; Liao, C.S. Benzoxazine as a reactive noncovalent dispersant for carbon nanotubes. RSC Adv. 2014, 4, 36012-36016. [CrossRef]

34. Lu, L.; Xing, D.; Xie, Y.; Teh, K.S.; Zhang, B.; Chen, S.; Tang, Y. Electrical conductivity investigation of a nonwoven fabric composed of carbonfibers and polypropylene/polyethylene core/sheath bicomponentfibers. Mater. Des. 2016, 112, 383-391. [CrossRef]

35. Sun, X.R.; Gong, T.; Pu, J.H.; Bao, R.-Y.; Xie, B.H.; Yang, M.B.; Yang, W. Effect of phase coarsening under melt annealing on the electrical performance of polymer composites with a double percolation structure. Chem. Chem. Phys. 2018, 20, 137-147. [CrossRef]

36. Jain, R.; Minus, M.L.; Chae, H.G.; Kumar, S. Processing, Structure, and Properties of PAN/MWNT Composite Fibers. Macromol. Mater. Eng. 2010, 295, 742-749. [CrossRef]

37. Bouchard, J.; Cayla, A.; Odent, S.; Lutz, V.; Devaux, E.; Campagne, C. Processing and characterization of polyethersulfone wet-spun nanocomposite fibres containing multiwalled carbon nanotubes. Synth. Met. 2016, 217, 304-313. [CrossRef]

(C) 2020 by the authors. Licensee MDPI, Basel, Switzerland. This article is an open access article distributed under the terms and conditions of the Creative Commons Attribution (CC BY) license (http://creativecommons.org/licenses/by/4.0/). 